\title{
Variabilidade espacial de atributos físico-químicos do solo e seus efeitos na produtividade do algodoeiro
}

\author{
Ronei S. Sana ${ }^{1}$, Ibanor Anghinoni ${ }^{2}$, Ziany N. Brandão ${ }^{3} \&$ Marquel J. Holzschuh $^{4}$ \\ ${ }^{1}$ SLC Agrícola SA, Porto Alegre, RS. E-mail: ronei.sana@slcagricola.com.br (Autor correspondente) \\ ${ }^{2}$ UFRGS, Porto Alegre, RS. E-mail: ibanghi@ufrgs.br \\ ${ }^{3}$ EMBRAPA/CNPA, Campina Grande, PB. E-mail: ziany.brandao@embrapa.br \\ ${ }^{4}$ SLC Agrícola SA, Porto Alegre, RS. E-mail: marquel.holzschuh@slcagricola.com.br
}

\section{Palavras-chave:}

agricultura de precisão

sensores de produtividade

condutividade elétrica aparente

\begin{abstract}
R E S U M O
No Brasil, a utilização de sensores de produtividade no algodoeiro é recente. A variabilidade de atributos físicos e químicos de solo abre a possibilidade de delimitar zonas homogêneas dentro da lavoura. Relações entre esses atributos e a produtividade do algodoeiro podem ser obtidas indicando o potencial da cultura em cada condição de solo. O objetivo deste trabalho foi estabelecer relações entre atributos de solo que determinem sua variabilidade espacial e que interferem na produtividade do algodoeiro. Foi conduzido um experimento em área de 56,1 ha, em Cristalina, GO, com a cultura do algodão. Amostragens de solo foram realizadas em uma grade regular de 80 x $80 \mathrm{~m}$ para avaliação dos atributos químicos e físicos e leituras contínuas de condutividade elétrica aparente (CEa). Os dados de produtividade do algodoeiro foram obtidos por colhedoras equipadas com sensor de fluxo, nas safras 2010/11 e 2011/12. A produtividade apresentou forte dependência espacial e possui correlação negativa com CEa, $\mathrm{pH} \mathrm{CaCl} ;$; $\mathrm{CEa}$ foi uma ferramenta adequada para identificação da variabilidade de acidez e de nutrientes no solo na condição estudada possibilitando delimitar zonas homogêneas, para o manejo de forma diferenciada.
\end{abstract}

Key words:

precision agriculture

yield mapping

apparent electrical conductivity

\section{Spatial variability of physical-chemical attributes of soil and its effects on cotton yield}

\begin{abstract}
A B S T R A C T
The use of cotton productivity sensors is recent in Brazil. Variability of physical and chemical properties of soil opens the possibility of delimiting homogeneous zones in crop fields. Relationships between these properties and cotton yield can be obtained, indicating the crop potential for each soil condition. The objective of this study was to establish relationships between soil attributes that have spatial variability and its interference in cotton yield. The experiment was carried out in 56.1 ha, in Cristalina, GO, with cotton. Soil samples were collected on a regular grid of $80 \times 80 \mathrm{~m}$ for evaluation of chemical and physical attributes and apparent electrical conductivity of soil (ECa). The cotton yield was measured by machines equipped with flow sensor during 2010/11 and 2011/12 cropping seasons. Cotton yield showed strong spatial dependence and has negative correlation with $\mathrm{ECa}$ and $\mathrm{pH} \mathrm{CaCl}_{2}$. The ECa was an useful tool to identify the variability of acidity and soil nutrients in this study, allowing to delimit homogeneous zones to a distinct management.
\end{abstract}

\section{INTRODUÇÃo}

Dentre as principais ferramentas utilizadas para identificação dos fatores que afetam o desenvolvimento das culturas, está a utilização de mapas de produtividade. Tais mapas são utilizados na agricultura de precisão (AP) como alternativa moderna visando gerenciar a variabilidade espacial e temporal de lavouras orientando práticas de manejo (Molin \& Rabelo, 2011; Milani et al., 2006; Blackmore et al., 2003). Os mapas de produtividade ilustram esta variabilidade (Guo et al., 2012). Na análise de Lark et al. (1999) a sequência de mapeamentos de rendimento é uma forma de definir unidades de manejo em um talhão associandoas aos atributos de solo.

A produção de algodão no cerrado brasileiro é bastante tecnificada e possui alto valor agregado. Esta cultura possui grande exigência nutricional principalmente com nitrogênio $(\mathrm{N})$, fósforo $(\mathrm{P})$ e potássio $(\mathrm{K})$; assim, a identificação da variabilidade dos atributos do solo e a geração de mapas de produtividade podem ser ferramentas importantes para identificar os problemas de manejo da cultura visando altas produtividades.

A utilização de sensores de produtividade no algodoeiro no Brasil é recente considerando-se que sua disponibilidade em áreas comerciais se iniciou na safra 2010/11 e ainda possui pequena adoção devido à falta de informações técnicas e ao custo de aquisição dos equipamentos. A produtividade é medida por sensores de fluxo de massa (densidade $\mathrm{x}$ velocidade) que ficam instalados junto aos dutos de entrada de algodão na colhedora e medem a quantidade que passa através dos mesmos sendo 
necessário um sistema de posicionamento global diferencial e um dispositivo de armazenamento de dados (Deere, 2012).

$\mathrm{Na}$ interpretação de um mapa de produtividade com a finalidade de gerenciamento da lavoura de algodão deve-se considerar, sobremaneira, as causas consistentes de variabilidade (Cerri \& Magalhães et al., 2012). Essas causas podem ser compreendidas pelo acompanhamento e análise dos possíveis fatores que influenciam a variabilidade durante safras sucessivas de vez que a variação espacial é resultado de um complexo de interações climáticas, edáficas, antrópicas e biológicas.

Tornou-se necessário um tempo maior para o desenvolvimento dos sensores de colhedoras de algodão, mas atualmente eles atingiram um nível aceitável de desvio, similar aos já alcançados pelos sensores de produtividade utilizados nas culturas de grãos que atuam atingem, atualmente, desvios ao redor de 2,0\% de erro (Konopatzki et al., 2012).

O conhecimento da variabilidade espacial dos atributos químicos e físicos do solo torna-se, então, importante para definir as práticas agronômicas a serem adotadas no algodoeiro desde que a produtividade se relacione com os atributos químicos, como acidez disponibilidade de nutrientes, e físicos, como granulometria e escassez ou excesso de água (Souza et al., 2001; Cerri \& Magalhães, 2012). Entre os atributos relacionados à disponibilidade de nutrientes $\mathrm{o} \mathrm{pH}$ do solo possui uma relação e, na maioria dos casos, apresenta a menor variabilidade espacial. Assim, é desejável que o $\mathrm{pH}$ não seja baixo e, consequentemente, a saturação por bases seja superior a $50 \%$, sendo que altas produtividades do algodoeiro são obtidas com saturação por bases na ordem de 60\% (Rosolem et al., 2012). O fósforo (P) disponível nos solos da região do Cerrado em condição natural é muito baixo; no entanto, nos solos cultivados a variação pode ser grande em razão da alta capacidade de retenção do $P$ na fase sólida e da sua aplicação na linha de semeadura. Nas classes Média/Alta de sua disponibilidade (Sousa et al., 2004) a resposta do algodoeiro à adição de $\mathrm{P}$ não ocorre necessariamente. Outros fatores, como a disponibilidade de água no solo, condicionam o rendimento da cultura cuja distribuição da precipitação durante o ciclo produtivo influencia diretamente a produtividade. Em condições de encharcamento do solo a produtividade do algodoeiro é reduzida (Souza et al., 2001).

Como alternativa prática, rápida e de baixo custo para mapear a variabilidade do solo, pode-se utilizar a condutividade elétrica aparente (CEa) uma medida da atividade iônica da solução do solo, a forma predominante de ocorrência dos nutrientes na sua fase líquida e de sua absorção pelas plantas. A CEa também tem relação com os atributos físicos, como umidade do solo, que é determinada pela textura, estrutura e a matéria orgânica (Molin \& Rabello, 2011). A CEa pode ser facilmente obtida no campo a partir de leituras com o sensor Veris 3100 e potencialmente utilizada para delimitar zonas homogêneas.

Objetivou-se com este trabalho avaliar a variabilidade espacial dos atributos químicos e físicos do solo e seus efeitos na produtividade do algodoeiro, como subsídio à agricultura de precisão.

\section{Material e Métodos}

Este trabalho foi desenvolvido em uma área comercial com 56,1 ha na Fazenda Pamplona, em Cristalina, GO, cujas coordenadas de referência são $16^{\circ} 13^{\prime} 40,15^{\prime \prime}$ S e $47^{\circ} 37^{\prime} 40,77^{\prime \prime}$ $\mathrm{O}$, com altitude média de $1010 \mathrm{~m}$, o solo foi classificado como Latossolo Vermelho-Amarelo distrófico (EMBRAPA, 2013). Na região central do experimento o solo é bem drenado e ao sul ocorre uma região mal-drenada (lençol freático raso). O clima da região, segundo a classificação de Köppen, é do tipo Tropical (Aw) com estação seca definida. A precipitação média anual é de $1516,1 \mathrm{~mm}$, no período de outubro a maio, sendo $1.308,3 \mathrm{~mm}$ na safra 2010/11,13,7\% inferior à média e, na safra 2011/12, a precipitação foi $1973 \mathrm{~mm}, 30,17 \%$ superior àquela média.

A área foi cultivada em sistema de semeadura direta desde 2001, em rotação de algodão (Gossypium hirsutum L.), soja (Glycine max L.) e milho (Zea mays) semeados no inicio das chuvas, entre outubro e novembro A última correção de acidez do solo com calcário foi realizada na safra 2010/11 com a aplicação de 2,2 $\mathrm{t} \mathrm{ha}^{-1} \mathrm{em}$ superfície. A adubação utilizada foi $150 \mathrm{~kg} \mathrm{ha}^{-1}$ de nitrogênio em cobertura, nas formas de sulfato de amônio (149 $\left.\mathrm{kg} \mathrm{ha}^{-1}\right)$ aos $41 \mathrm{DAE}$, duas aplicações de ureia, 141 e $145 \mathrm{~kg} \mathrm{ha}^{-1}$, aos 63 e 100 DAE, respectivamente, $120 \mathrm{~kg}$ ha $^{-1} \mathrm{de}_{2} \mathrm{O}_{5}$, na forma de fertilizante formulado (05-32-00) e 120 $\mathrm{kg} \mathrm{ha}^{-1}$ de $\mathrm{K}_{2} \mathrm{O}$ com a aplicação de cloreto de potássio. Foram utilizadas as cultivares FMT 701, na safra 2010/11 e Fiber Max 993, na safra 2011/12, com espaçamento entre linhas de 0,76 m.

Antes da semeadura da safra 2011/12 foram implantadas, na área experimental, diferentes doses de $\mathrm{P}_{2} \mathrm{O}_{5}$ (com o formulado 05-32-00) visando verificar a resposta do algodoeiro à adubação fosfatada e estabelecidas duas repetições para cada dose, 0,60 , $120,180,240$ e $300 \mathrm{~kg} \mathrm{ha}^{-1}$ de $\mathrm{P}_{2} \mathrm{O}_{5}$, em que a dose $0 \mathrm{~kg} \mathrm{ha}^{-1}$ identifica a testemunha, $60 \mathrm{~kg} \mathrm{ha}^{-1}$ referentes à meia dose da adubação padrão utilizada na lavoura, $120 \mathrm{~kg} \mathrm{ha}^{-1}$ representam a dose padrão variando a dose até duas doses e meia padrão. As doses de $\mathrm{N}$ também variaram nos tratamentos $0,9,19,28$, 37 e $47 \mathrm{~kg} \mathrm{ha}^{-1}$, respectivamente.

Foram demarcados 90 pontos na área experimental espaçados em grade amostral de $80 \times 80 \mathrm{~m}$, representando 0,64 hectares por amostra composta por 7 subamostras coletadas em um raio de até $10 \mathrm{~m}$ e profundidade de 0-0,2 m. Utilizouse, para isto, um aparelho de GPS modelo 76CSx da Garmin. Esses pontos foram utilizados como referência para todas as amostragens, medições e leituras. As análises seguiram metodologia da EMBRAPA (2009) para extração de $\mathrm{Ca}^{2+}, \mathrm{Mg}^{2+}$ e $\mathrm{K}^{+}$trocáveis e $\mathrm{pH}$ em água e $\mathrm{pH}$ em $\mathrm{CaCl}_{2}$. Os resultados de soma de bases (SB), saturação de bases (V\%) e CTC, foram calculados a partir dos dados de $\mathrm{Ca}, \mathrm{Mg}, \mathrm{K}$, e $\mathrm{H}+\mathrm{Al}$. A matéria orgânica foi determinada pelo método da oxidação; o fósforo foi extraído pelos métodos: Mehlich-1 e resina trocadora de ânions (RTA). As frações granulométricas foram determinadas pelo método de dispersão total.

A condutividade elétrica aparente (CEa) foi determinada 20 dias antes da semeadura da safra 2010/11. Utilizou-se o equipamento modelo Veris 3000 (VERIS, 2011) percorrendo a área experimental em condição úmida coletando os dados 
em intervalos de um segundo e as coordenadas registradas por GPS Trimble.

$\mathrm{Na}$ safra 2010/11 a produtividade do algodoeiro foi determinada utilizando-se a colhedora de algodão modelo John Deere 9996 (Deere, 2012) equipada com o sistema GreenStar Harvest Doc ${ }^{\oplus}$, composto de sensor de rendimento do tipo fluxo ultrassônico, monitor de dados (Monitor GreenStar" ${ }^{\mathrm{mm}}$ ), cartão de armazenamento de dados (Keycard) e antena receptora de Sistema de Posicionamento Global (GPS) com sinal diferencial, receptor StarFire ${ }^{\mathrm{m} w}$; na safra 2011/12 a colhedora utilizada foi do modelo John Deere 7760 mantendo-se o mesmo conjunto de mensuração da produtividade (Figura 1).

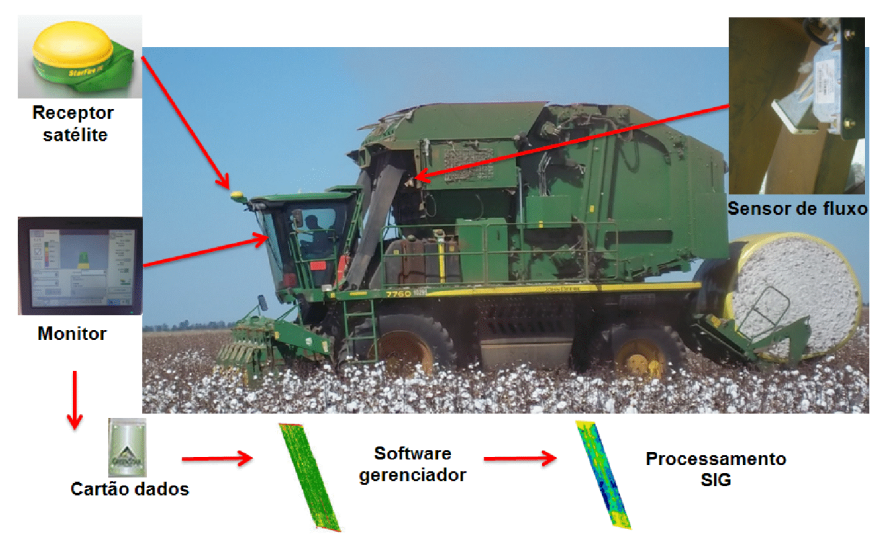

Figura 1. Representação dos componentes necessários para mensuração da produtividade no algodão

O sensor de fluxo de massa, localizado ao final do duto de sucção da colhedora, capta dados a cada $3 \mathrm{~s}$. A calibração dos sensores foi realizada pela pesagem do equipamento transbordo que recebe o volume colhido pela colhedora em balança rodoviária, sendo este o fator de calibração utilizado durante a colheita. Os dados brutos foram descarregados no software Apex (John Deere, 2012) e convertidos no formato Shapefile e Excel (.xls) para o tratamento e processamento das informações, limpeza de erros e filtragem de dados. A limpeza se deu devidoà ocorrência de pontos com ausência de coordenadas ou externos à bordadura da lavoura, além de erros de manobras e paradas da máquina (Menegatti \& Molin, 2004).

Os dados foram submetidos à análise da estatística descritiva e à geoestatística (Vieira, 2000) para determinação da variabilidade espacial. Semivariogramas foram construídos com base no cálculo da semivariância $\gamma(\mathrm{h})$ por meio da seguinte expressão:

$$
\gamma(\mathrm{h})=\frac{1}{2 \mathrm{~N}} \sum_{\mathrm{i}=1}^{\mathrm{N}(\mathrm{h})}\left[\mathrm{Z}\left(\mathrm{X}_{\mathrm{i}}\right)-\mathrm{Z}\left(\mathrm{X}_{\mathrm{i}+\mathrm{h}}\right)\right]^{2}
$$

em que:

$\gamma(\mathrm{h})$ - semivariância estimada a partir de dados experimentais $\mathrm{N}(\mathrm{h})$ - número de pares de valores medidos na distância, $\mathrm{h}$

$\mathrm{Z}\left(\mathrm{X}_{\mathrm{i}}\right)$ - valor da variável medida na posição $\mathrm{x}_{\mathrm{i}}$,

$\mathrm{Z}\left({ }_{\mathrm{xi}+\mathrm{h}}\right)$ - valor da variável medida na posição $x\left({ }_{\mathrm{i}+\mathrm{h}}\right)$

i - posição da amostra

h - distância entre duas amostras
Foram testados os modelos matemáticos linear, esférico, gaussiano e exponencial para ajustes do semivariograma experimental pelo software SGeMS (SGeMS-ar2tech-betawin32-2012). Para a análise de tendência dos dados foi utilizada a técnica de "Jack kniffing" (Vieira, 2000) e os semivariogramas experimentais foram considerados isotrópicos. Para caracterizar a dependência espacial dos atributos de solo foram testados o Índice de Dependência Espacial (IDE) proposto por Cambardella et al. (1994) classificando, como forte, o IDE < $25 \%$, IDE moderado entre 25 e $75 \%$ e fraco com IDE $>75 \%$. A equação é definida por:

$$
\mathrm{IDE}=100 \frac{\mathrm{C}_{0}}{\mathrm{C}_{0}+\mathrm{C}_{1}}
$$

em que:

$\mathrm{C}_{0}$ - efeito pepita

$\mathrm{C}_{0}+\mathrm{C}_{1}$ - patamar

Após verificada a dependência espacial para os atributos químicos e físicos do solo os dados foram interpolados utilizando-se semivariogramas das variáveis amostradas, pelo método da krigagem como técnica de estimação. No tratamento dos dados de produtividade o método de interpolação foi o inverso do quadrado da distância (Amado et al., 2007). Os mapas foram gerados no software SSToolBox, versão 2009.

Foi realizada análise de correlação dos atributos químicos e físicos de solo obtidos pela análise de cada ponto da grade amostral e a produtividade média das leituras do sensor obtidas próximo a cada ponto amostral com uso do coeficiente de correlação simples, a correlação de Pearson $(\mathrm{p}<0,01)$. Os dados foram submetidos à análise estatística descritiva com base na metodologia utilizada por Cambardella et al. (1994), em três estágios: 1) distribuição das frequências e testes de normalidade; 2) distribuições descritas por métodos estatísticos tradicionais (média, desvio padrão e coeficiente de correlação) e 3) semivariogramas com efeito pepita e alcance das variáveis.

\section{Resultados e Discussão}

Os atributos de solo apresentaram dados de distribuição variáveis. Os teores de Ca e $\mathrm{K}$ trocáveis e de $\mathrm{P}$ disponível (Mehlich-1) apresentaram os maiores valores de assimetria e curtose (Tabela 1). Para os coeficientes de variação (CV) menores que $35 \%$, a série de dados é considerada homogênea e a média representa o conjunto de dados; quando maiores que $35 \%$, indicam que a série é considerada heterogênea e, se o CV for maior do que $65 \%$, a série é muito heterogênea e a média perde o seu significado (Vanni, 1998). Assim, o CV muito alto encontrado para P Mehlich-1 (74\%) indica série muito heterogênea e para P resina (50\%) a série é heterogênea (Tabela 1). Esta alta variação é determinada pelo histórico de manejo, correção, adubação do solo e pela mobilidade baixa do $\mathrm{P}$ em solo argiloso. Para os atributos $\mathrm{pH} \mathrm{CaCl}, \mathrm{pH}_{2} \mathrm{H}_{2} \mathrm{O}, \mathrm{Ca}, \mathrm{Mg}$ e K 
Tabela 1. Estatística descritiva dos atributos físico-químicos do solo e produtividade do algodoeiro

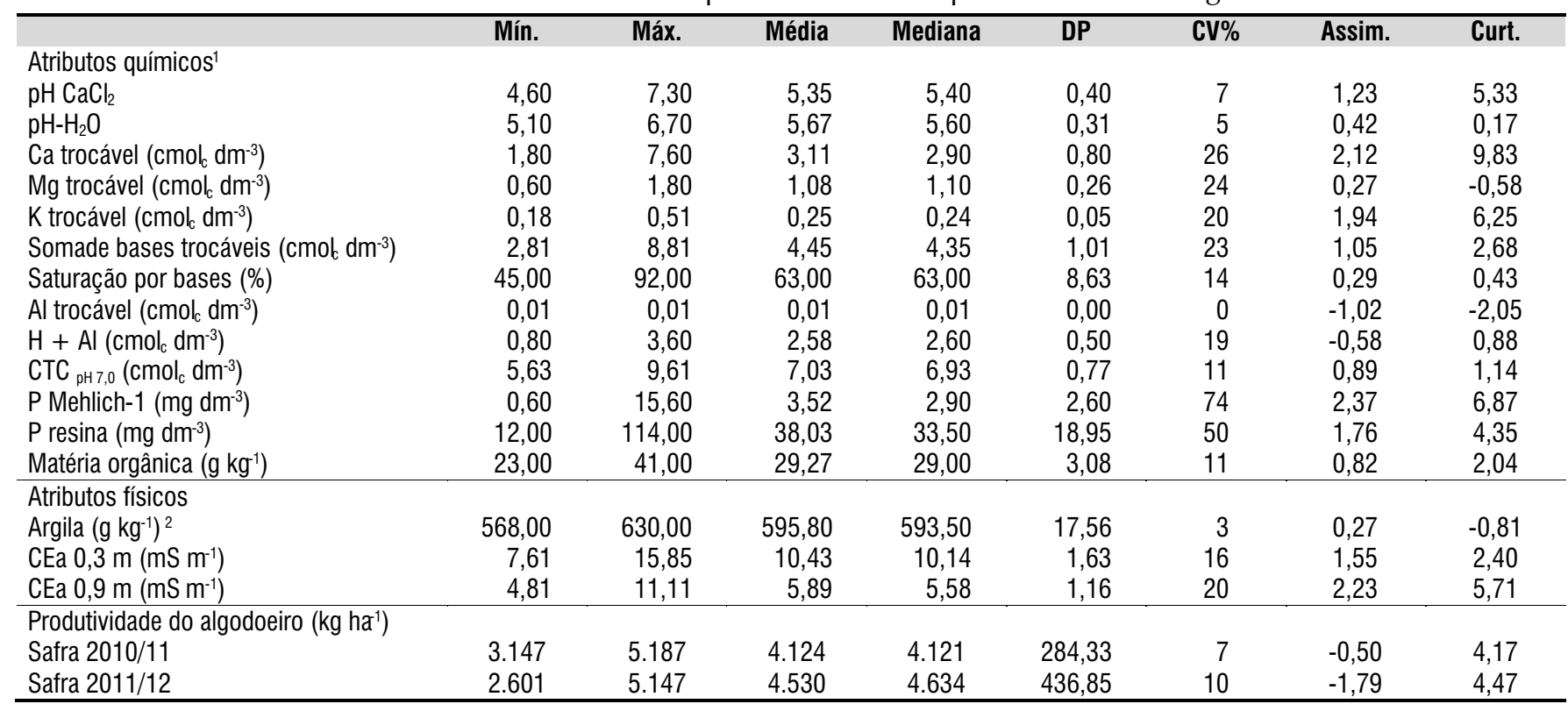

Mín. - Mínimo; Máx. - Máximo; DP - Desvio padrão; CV - Coeficiente de variação (\%); Assim. - Assimetria; Curt. = Curtose.

${ }^{1} \mathrm{pH} \mathrm{H}_{2} \mathrm{O}$ (1:1); $\mathrm{pH} \mathrm{CaCl}_{2}$ (1:1); pH SMP (10:10:5); $\mathrm{Ca}^{2+}, \mathrm{Mg}^{2+}$ e K+ trocáveis - Extração com resina trocadora de cátions - RTC; Matéria orgânica foi obtida pelo método da oxidação; $P$ Resina - Resina trocadora de ânions-RTA; P Mehlich-1 - Extraído por solução Mehlich-1 (EMBRAPA, 2009).

${ }^{2}$ Método de dispersão total, separando as frações areia, silte e argila.

trocáveis (Tabela 1) a variação foi homogênea sinalizando que a intensidade de amostragem de 0,64 amostras ha ${ }^{-1}$ foi adequada para representar a área.

Os atributos físicos avaliados apresentaram baixa variabilidade para a argila (Tabela 1) em que o CV encontrado foi entre os menores confirmando a pequena variação dos dados na área experimental, que é localizada em relevo plano. A CEa medida na profundidade de 0-0,3 m apresentou uma série de dados com menor variação em relação à CEa medida de $0-0,9 \mathrm{~m}$ mas ambas com CV classificados homogêneos. Pode ser visualizado (Figura 2) o mapa de $\mathrm{pH} \mathrm{CaCl}_{2}$ (Figura 2A) que apresentou similaridade em algumas regiões com o mapa de soma de bases trocáveis (Figura $2 \mathrm{~B}$ ) principalmente nos extremos sul e norte da área experimental. Pode-se identificar que entre o $\mathrm{pH} \mathrm{CaCl}_{2}$ e a CEa houve o mesmo comportamento nas diferentes zonas dos mapas, tanto na medida superficial (Figura 2D) como na subsuperficial (Figura 2E).

O mapa de P Mehlich-1 (Figura 2C) indica teores no solo muito baixos $\left(<2,0 \mathrm{mg} \mathrm{dm}^{-3}\right)$ e baixos $\left(2,1 \mathrm{a} 3,0 \mathrm{mg} \mathrm{dm}^{-3}\right)$ que representam $35,9 \%$ da área e, se considerado também o nível médio $\left(3,1 \mathrm{a} 4 \mathrm{mg} \mathrm{dm}^{-3}\right)$ representará $74,2 \%$ da área total (Figura 2), sendo que a faixa de suficiência para este solo é $>4,1 \mathrm{mg}$ $\mathrm{dm}^{-3}$ (Sousa et al., 2004).

Os semivariogramas obtidos para os atributos de solo foram ajustados melhor ao modelo esférico (Tabela 2) com exceção ao $P$ resina, que apresentou efeito pepita puro (EPP) o qual reflete a variabilidade existente em pequenas distâncias e também incorpora erros e ainda exigirá uma grade amostral mais densa para sua representação. O número mínimo e a qualidade das subamostras de solo por meio de amostragens densas visam garantir a malha amostral mais representativa de todos os atributos de solo que, neste estudo, é limitada pelo P (Vieira, 2000; Zonta et al., 2014).
Os atributos de solo $\mathrm{pH} \mathrm{H}_{2} \mathrm{O}$, CEa rasa, CEa profunda e argila, foram classificados com IDE Forte indicando haver alta relação entre as amostras. Para os demais atributos químicos se obtiveram IDE Moderado indicando que é possível estimar o comportamento desses atributos com a grade amostral utilizada. $\mathrm{O} \mathrm{pH} \mathrm{CaCl}_{2}$, apesar de ser classificado com IDE Moderado, apresenta boa similaridade espacial com a CEa. A determinação do alcance do semivariograma permite inferir sobre a máxima distância de homogeneidade representada em relação às amostras vizinhas. Dentre todos os atributos avaliados o P apresentou a menor representação espacial devido ao EPP encontrado no $\mathrm{P}$ resina e ainda pelo menor alcance entre os atributos medidos, que foi de $102 \mathrm{~m}$ para P Mehlich-1. Este comportamento é devido à sobreposição de problemas na amostragem do solo, à determinação laboratorial de $\mathrm{P}$, à retenção do nutriente ao solo e à forma de aplicação em linha (Sousa et al., 2004; Zonta et al., 2014).

Os dados de produtividade das safras 2010/11 e 2011/12 apresentaram baixo CV, de 7 e 10\%, respectivamente (Tabela 1). Referida variação está em acordo com as obtidas em outras culturas no Brasil, como soja, milho e trigo (Amado et al., 2007; Montezano, et al., 2006). Por outro lado, em estudo realizado em oito lavouras de algodão, no estado do Texas, EUA, o CV da produtividade de algodoeiro variou de 8 a 32\% (Guo et al., 2012) com menor homogeneidade do que a do presente trabalho. A amplitude entre a produtividade mínima e máxima na safra 2010/11 foi de $2.040 \mathrm{~kg} \mathrm{ha}^{-1}$ e na safra 2011/12, de $2.546 \mathrm{~kg} \mathrm{ha}^{-1}$ de algodão em caroço. Essas diferenças entre os dois anos são devidas à distribuição irregular das plantas na lavoura na safra 2011/12 ocasionada pelo excesso de chuva, além das diferentes doses de $\mathrm{N}$ e $\mathrm{P}$ aplicados na área experimental

A quantidade total de registros realizados pelo sensor da máquina foi de 1.233 pontos ha ${ }^{-1}$; quando se comparam, aos 
A.

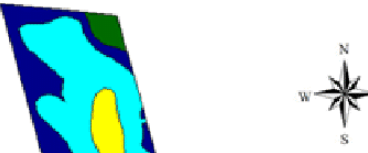

B.

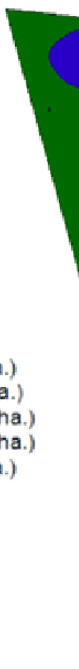

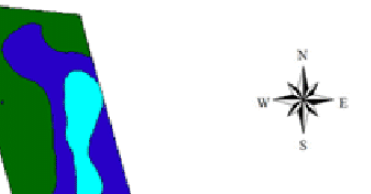

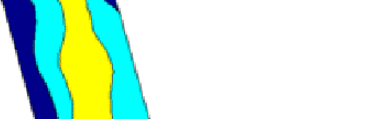

$\checkmark$

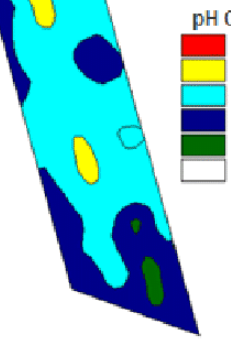

D.
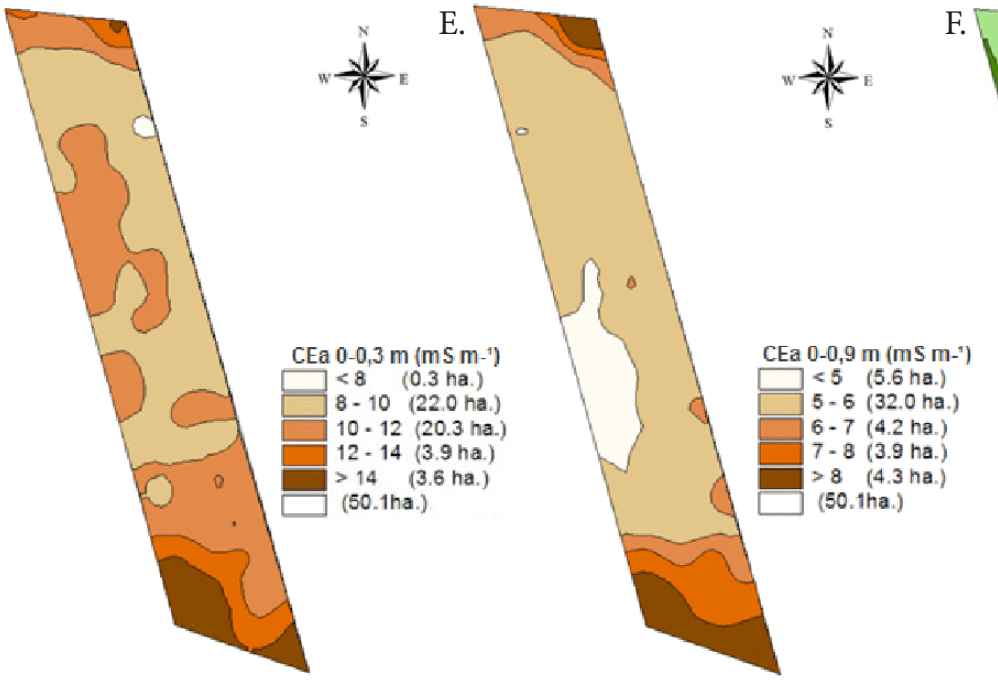

C.

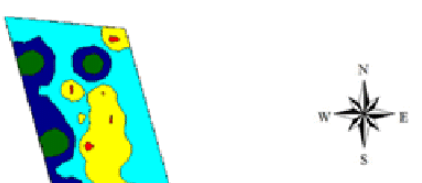

Soma Bases ( $\left.\mathrm{cmol}_{5} \mathrm{dm}_{-}{ }^{-}\right)$ $<1,6 \quad(0.0 \mathrm{ha}$ ) $1.6-2.5$ (0.0 ha.)
$2.5-3.5(5.1 \mathrm{ha})$ $3.5-4.5$ (22.9 ha.) $>4,5$ (22.1 ha.) (50.1ha.) $5,6=6,0 \quad(13.2 \mathrm{~h}$ (50.1ha.)

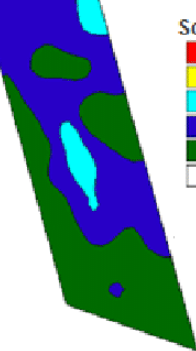

(2)
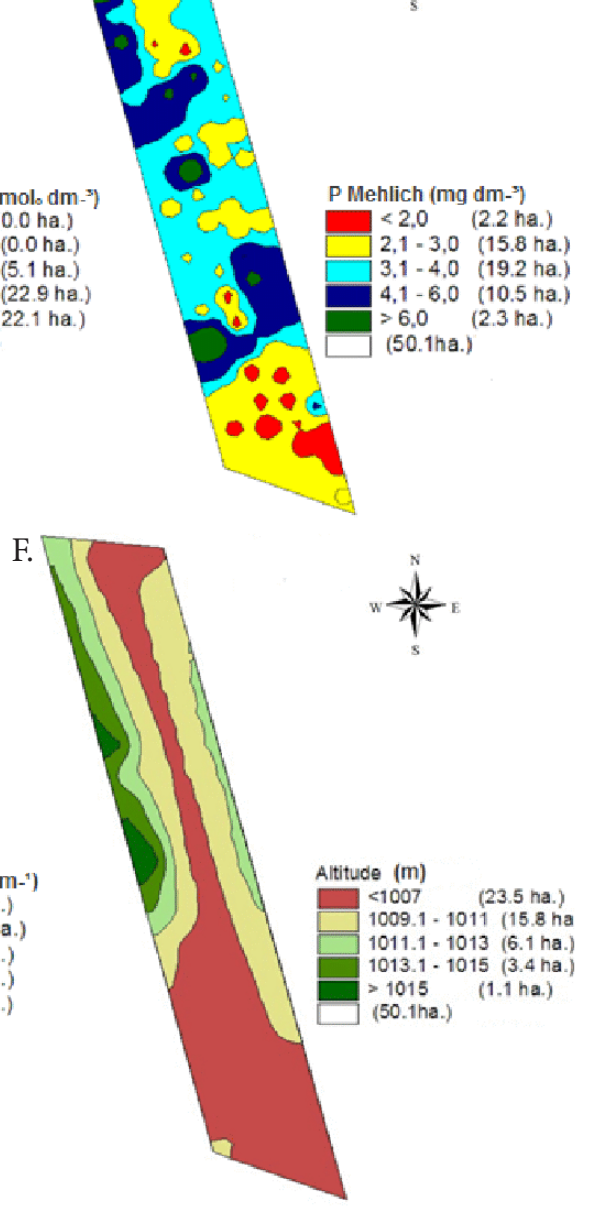

Obs.: Amostras e leituras realizadas antes da semeadura do algodão, na safra 2010/11

Figura 2. Mapas de superfície de atributos: $\mathrm{pH} \mathrm{CaCl}_{2}(\mathrm{~A})$; Soma de bases trocáveis (B); $\mathrm{P}$ Mehlich-1(C); CEa rasa (0$0,3 \mathrm{~m})(\mathrm{D}) ;$ CEa profunda $(0-0,9 \mathrm{~m})(\mathrm{E})$; Altitude(F)

Tabela 2. Componentes da semivariância e Índice de Dependência Espacial (IDE) dos atributos químicos e físicos do solo e da produtividade do algodoeiro

\begin{tabular}{|c|c|c|c|c|c|c|c|}
\hline & \multirow{2}{*}{ Modelo } & \multicolumn{3}{|c|}{ Semivariância } & \multirow{2}{*}{$\begin{array}{l}\text { Alcance } \\
\text { (A) }\end{array}$} & \multirow{2}{*}{$\begin{array}{c}\text { IDE } \\
\left(C_{0} /\left(C_{0}+C_{1}\right)\right) * 100\end{array}$} & \multirow{2}{*}{ Classe } \\
\hline & & $C_{0}$ & $C_{1}$ & $\mathrm{C}_{0}+\mathrm{C}_{1}$ & & & \\
\hline \multicolumn{8}{|l|}{ Atributos químicos } \\
\hline $\mathrm{pH} \mathrm{H}_{2}$ & $\begin{array}{l}\text { Esserico } \\
\text { Esférico }\end{array}$ & $\begin{array}{l}0,05 \\
0,02\end{array}$ & 0,08 & $\begin{array}{l}0,10 \\
010\end{array}$ & $\begin{array}{l}255 \\
301\end{array}$ & $\begin{array}{l}31 \\
20\end{array}$ & MI \\
\hline Ca trocável & Esférico & 0,20 & 0,45 & 0,65 & 206 & 31 & M \\
\hline Mg trocável & Esférico & 0,02 & 0,04 & 0,06 & 206 & 33 & M \\
\hline K trocável & Esférico & 0,01 & 0,02 & 0,03 & 283 & 33 & M \\
\hline SB trocáveis & Esférico & 0,40 & 0,45 & 0,85 & 211 & 47 & M \\
\hline Saturação bases & Esférico & 25,00 & 40,00 & 65,00 & 210 & 38 & M \\
\hline $\mathrm{H}+\mathrm{Al}$ trocáveis & Esférico & 0,09 & 0,14 & 0,23 & 260 & 39 & $\mathrm{M}$ \\
\hline $\mathrm{CTC}_{\mathrm{pH} 7,0}$ & Esférico & 0,25 & 0,35 & 0,60 & 203 & 42 & M \\
\hline P Mehlich 1 & Esférico & 4,00 & 5,00 & 9,00 & 102 & 44 & M \\
\hline P resina & EPP & - & - & - & - & - & - \\
\hline \multicolumn{8}{|l|}{ Atributos físicos } \\
\hline Argila & Esférico & 100,00 & 300,00 & 400,00 & 313 & 25 & $\mathrm{~F}$ \\
\hline CEa $(0-0,3 \mathrm{~m})$ & Esférico & 0,30 & 2,00 & 2,30 & 325 & 13 & $\mathrm{~F}$ \\
\hline CEa $(0-0,9 \mathrm{~m})$ & Esférico & 0,15 & 0,95 & 1,10 & 308 & 14 & $\mathrm{~F}$ \\
\hline \multicolumn{8}{|l|}{ Produtividade } \\
\hline Prod. 2010/11 & Gaussiano & 60000 & 45000 & 105000 & 410 & 57,14 & M \\
\hline Prod. 2011/12 & Esférico & 20000 & 175000 & 195000 & 648 & 10,26 & $\mathrm{~F}$ \\
\hline
\end{tabular}

$\mathrm{C}_{0}$. Efeito pepita; $\mathrm{C}_{1}$ - Contribuição; $\mathrm{C}_{0}+\mathrm{C}_{1}$ - Patamar; Classe: $\mathrm{F}$ - Forte, $\mathrm{M}$ - Moderada 
mapas obtidos na colheita de outras culturas, o número de pontos registrados é menor; no caso do milho foram somente 580 pontos ha ${ }^{-1}$ (Michelan et al., 2007) e 863 pontos ha ${ }^{-1}$ (Menegatti \& Molin, 2004) para a soja, Amado et al. (2007) obtiveram entre 156 e 401 pontos ha ${ }^{-1}$, em diferentes safras e áreas de soja; desta forma, a colheita de algodão utilizando sensores de produtividade permite investigar a microvariabilidade pela maior quantidade e qualidade dos pontos registrados, cada ponto representando uma área útil de $8,1 \mathrm{~m}^{2}$. A colhedora utiliza todas as linhas de cultivo diferentemente das plataformas de grãos que dependem da habilidade do operador para indicar a largura efetiva de colheita. Outras características do sensor são a não existência de contato direto com o produto, dispensando limpeza periódica, e a inexistência de sensor de umidade em virtude da mensuração ser realizada por fluxo, e a variação da umidade ao longo do dia não interferir no fluxo, diferente da colheita de grãos em que o sensor é outro fator de incerteza.

A produtividade obtida na colheita com sensor em relação à obtida na balança, com a pesagem dos fardos, foi 8,4\% inferior na safra 2010/11 e 3,2\% superior na safra 2011/12. Essas variações podem estar associadas às calibrações realizadas nas máquinas tal como pela época de colheita, quanto mais tardia ocorre o escorrimento do algodão no capulho. A produtividade obtida pelo sensor tende a ser superestimada quando a produtividade da lavoura é alta, como exemplo, na variedade Fiber Max 993 (safra 2011/12) e subestimada quando a produtividade da lavoura é baixa, casos em que é aconselhável realizar a calibração, sempre ao trocar de lavoura e, indispensavelmente, ao mudar de cultivar. De acordo com Blackmore et al. (2003) o mapa de produtividade da cultura é o melhor indicador entre todos os fatores que influenciam o rendimento; entretanto, a coleta de dados pela colhedora deve ser acompanhada de cuidados operacionais, como a largura efetiva de linhas na plataforma, entrada e saída da lavoura, número de manobras.

A produtividade apresentou, quando submetida aos modelos de semivariogramas, melhor ajuste ao gaussiano e esférico nas safras 2010/11 e 2011/12 respectivamente (Tabela 2). Na safra 2010/11 o mapa resultante da utilização de sensores de produtividade apresentou zona homogênea na faixa de 3500 a $4000 \mathrm{~kg} \mathrm{ha}^{-1}$ (Figura 3A). Este comportamento pode ser justificado em razão da falha de alguns registros de dados pelo conjunto de armazenamento, instalado na máquina. Em 57,7\% da área ocorreu produtividade $\geq$ de $4.000 \mathrm{~kg} \mathrm{ha}^{-1}$ de algodão em caroço, considerada satisfatória.

A produtividade obtida na safra 2011/12 (Figura 3C) demonstra grande variabilidade espacial de vez que foi influenciada pelas diferentes doses de $\mathrm{P}_{2} \mathrm{O}_{5}$ antes aplicadas à semeadura (Figura $3 \mathrm{~B}$ ) indicando que houve sensibilidade para identificar as zonas com diferentes doses de fósforo e ao efeito do $\mathrm{N}$ adicionado ao formulado 05-32-00. O mapa possibilitou detectar produtividades inferiores à média da lavoura nas extremidades sul e norte (Figura 3C); somente 10,5\% da área apresentaram produtividade inferior a $4.000 \mathrm{~kg} \mathrm{ha}^{-1}$ enquanto sua distribuição se concentrou nas extremidades norte e sul da área experimental e no eixo central longitudinal, exatamente onde não houve adição de $\mathrm{P}_{2} \mathrm{O}_{5}$ (Figura 3B).

A investigação dos fatores que afetam a produtividade das culturas deve ser iniciada com o estudo do banco de dados de histórico de manejo, adubações, rotação de culturas e produtividade obtida em cada lavoura. Quando é identificado comportamento heterogêneo, pode-se efetuar aplicações de fertilizantes e corretivos de acidez em taxa variável e também realizar manejos regionalizados de solo e da cultura (Torbett et al., 2007). Entretanto, quando os fatores que afetam a produtividade não podem ser corrigidos devido às características intrínsecas do solo é aconselhável utilizar a estratégia de criação de zonas de manejo e tratá-las diferentemente ajustando as tecnologias conforme o potencial produtivo de cada local (Walton et al., 2008). O mapa de produtividade demonstrou zonas com diferentes potenciais produtivos que podem ser isolados em especial nas áreas sul e norte do mapa. A correção da fertilidade e a adaptação de manejos são decisões que devem ser realizadas.
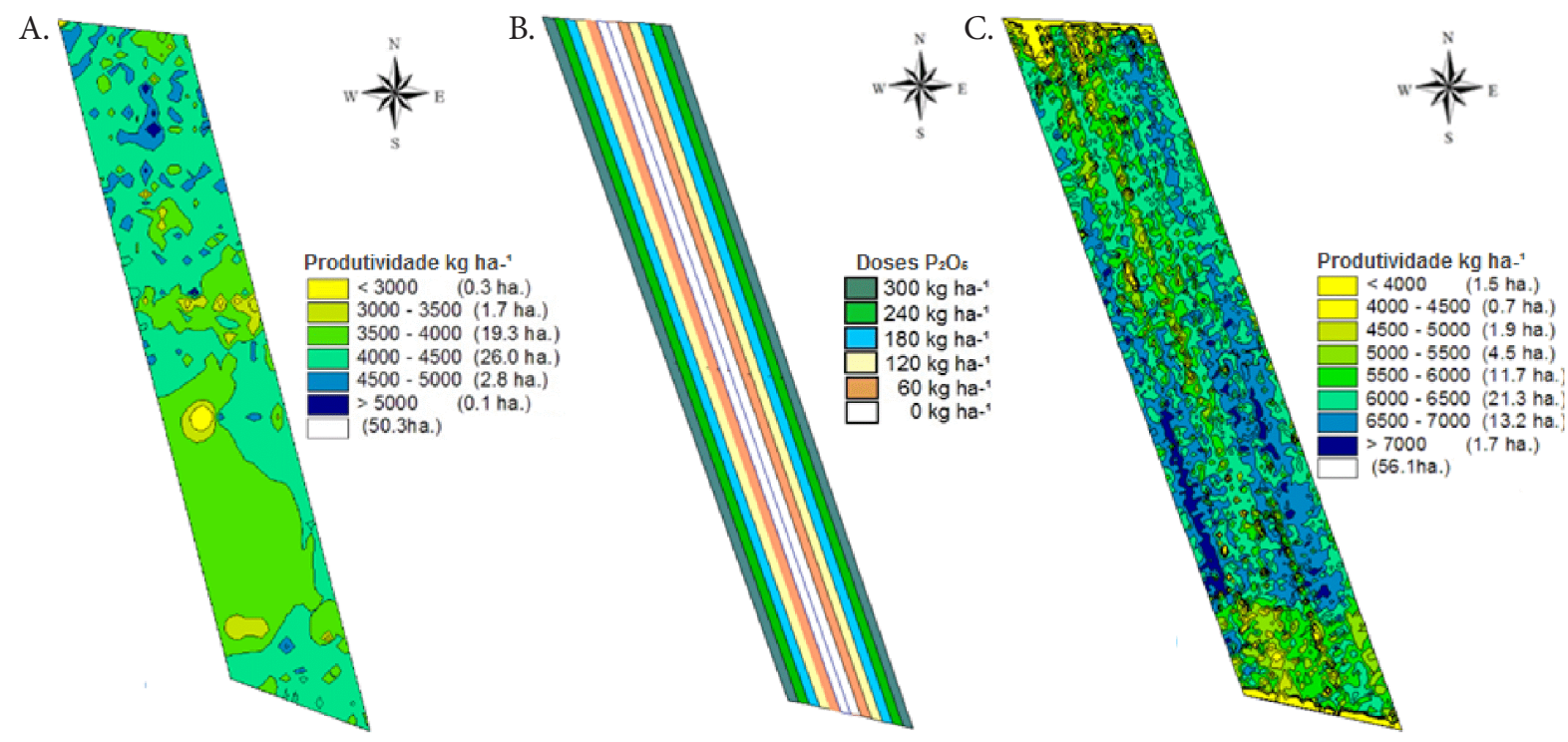

Figura 3. Variação espacial da produtividade de algodoeiro na safra 2010/11 (A); Doses de $\mathrm{P}_{2} \mathrm{O}_{5}$ aplicadas antes da semeadura da safra 2011/12 (B); Variação espacial da produtividade de algodoeiro na safra 2011/12 (C) 
Tabela 3. Coeficientes de correlação entre os atributos químicos e físicos do solo e produtividade do algodoeiro, nas safras 2010/11 e 2011/12

\begin{tabular}{|c|c|c|c|c|c|c|c|c|c|c|c|c|c|c|c|}
\hline & $\begin{array}{l}\text { Prod. } \\
10 / 11\end{array}$ & $\begin{array}{l}\text { Prod. } \\
11 / 12\end{array}$ & $\begin{array}{r}\mathrm{CE} \\
(0.3 \mathrm{~m})\end{array}$ & $\mathrm{a}$ & Argila & $\mathrm{pH} \mathrm{CaCl}{ }_{2}$ & $\mathrm{pH} \mathrm{H} \mathrm{H}_{2} \mathrm{O}$ & $\mathbf{H}+\mathbf{A l}$ & $\mathrm{Ca}$ & Mg & K & CTC $_{\text {pH 7,0 }}$ & V\% & P Mehlich-1 & $P$ resina \\
\hline Prod. 11/12 & $-0,11$ & & & & & & & & & & & & & & \\
\hline $\mathrm{CEa}(0,3 \mathrm{~m})$ & $-0,19$ & $-0,50^{*}$ & - & & & & & & & & & & & & \\
\hline CEa $(0,9 \mathrm{~m})$ & $-0,13$ & $-0,55^{*}$ & $0,83^{*}$ & - & & & & & & & & & & & \\
\hline Argila & 0,20 & 0,24 & $-0,25$ & $-0,20$ & - & & & & & & & & & & \\
\hline $\mathrm{pH} \mathrm{CaCl}{ }_{2}$ & 0,10 & $-0,28^{*}$ & $0,42^{*}$ & $0,58^{*}$ & $-0,08$ & - & & & & & & & & & \\
\hline $\mathrm{pH} \mathrm{H}_{2} \mathrm{O}$ & 0,12 & $-0,43^{\star}$ & $0,60^{*}$ & $0,53^{*}$ & $-0,16$ & $0,52^{*}$ & - & & & & & & & & \\
\hline $\mathrm{H}+\mathrm{Al}$ & 0,02 & $0,27^{*}$ & $-0,58^{*}$ & $-0,68^{\star}$ & 0,18 & $-0,89^{*}$ & $-0,59^{*}$ & - & & & & & & & \\
\hline $\mathrm{Ca}$ & 0,04 & $-0,41^{\star}$ & $0,46^{*}$ & $0,56^{*}$ & $-0,15$ & $0,74^{\star}$ & $0,54^{*}$ & $-0,63^{*}$ & & & & & & & \\
\hline $\mathrm{Mg}$ & 0,16 & $-0,24$ & $0,33^{*}$ & $0,30^{*}$ & $-0,09$ & $0,61^{*}$ & $0,51^{*}$ & $-0,60^{\star}$ & $0,65^{\star}$ & - & & & & & \\
\hline K & 0,01 & $-0,22$ & $0,48^{*}$ & $0,30^{*}$ & $-0,05$ & $0,38^{*}$ & $0,33^{\star}$ & $-0,42^{\star}$ & 0,30 * & $0,45^{\star}$ & - & & & & \\
\hline $\mathrm{CTC}_{\mathrm{pH} 7,0}$ & 0,11 & $-0,34^{\star}$ & 0,26 & $0,28^{*}$ & $-0,07$ & $0,42^{*}$ & $0,38^{*}$ & $-0,24$ & $0,88^{*}$ & $0,66^{*}$ & 0,26 & - & & & \\
\hline & 0,05 & $-0,36^{*}$ & $0,56^{*}$ & $0,62^{*}$ & $-0,19$ & $0,88^{*}$ & $0,63^{\star}$ & $-0,90^{*}$ & $0,85^{\star}$ & $0,81^{*}$ & $0,48^{\star}$ & $0,61^{*}$ & - & & \\
\hline P Mehlich-1 & 0,09 & 0,11 & $-0,21$ & $-0,26$ & 0,03 & $-0,08$ & $-0,15$ & 0,20 & 0,04 & 0,15 & $-0,02$ & 0,22 & $-0,05$ & - & \\
\hline$P$ resina & $-0,02$ & 0,05 & 0,01 & 0,00 & $-0,06$ & 0,06 & $-0,08$ & 0,02 & 0,16 & 0,21 & 0,13 & 0,26 & 0,11 & $0,90^{*}$ & - \\
\hline MOS & 0,05 & $-0,04$ & 0,00 & $-0,13$ & 0,00 & 0,12 & 0,11 & $-0,05$ & 0,20 & $0,29^{*}$ & 0,14 & $0,29^{*}$ & 0,19 & 0,13 & 0,08 \\
\hline
\end{tabular}

*Correlação Pearson; Significativo a 1\% de probabilidade pelo teste t; MOS - Matéria orgânica do solo; Prod. - Produtividade

Os atributos de solo e a produtividade foram correlacionados utilizando-se o coeficiente de correlação simples ( $\mathrm{p}<0,01)$; na safra 2010/11 a produtividade do algodoeiro não apresentou correlação com os atributos químicos e físicos do solo (Tabela 3) indicando que para, safra os atributos avaliados não justificaram a variação espacial e, possivelmente, a influência de outras variáveis afetou a produtividade, como a população de plantas, a incidência de pragas, doenças e ervas daninhas. Montezano et al. (2006) também não encontraram correlação entre a produtividade do milho e o teor de $\mathrm{P}, \mathrm{S}, \mathrm{K}, \mathrm{Ca}$ e $\mathrm{Mg}$ disponíveis no solo; na safra 2011/12 a produtividade teve correlação negativa com a CEa 0-0,3 m, CEa 0-0,9 m, pH CaCl, $\mathrm{pH} \mathrm{H}_{2} \mathrm{O}$, Ca trocável, CTC- ${ }_{p H 7,0}$ e saturação por bases, mesmo em condições de maior disponibilidade de nutrientes. Efeito similar foi observado por Cerri \& Magalhães et al. (2012) estudando a correlação entre os atributos químicos e a produtividade de cana-de-açúcar.

Foram observadas, durante o ciclo da cultura, regiões com acúmulo de água, com plantas de maior desenvolvimento vegetativo e com menor desenvolvimento reprodutivo. $\mathrm{Na}$ Figura $2 \mathrm{~F}$ podem ser visualizadas regiões com menores cotas altimétricas, principalmente ao sul, onde ocorrem períodos de encharcamento e o solo é visualmente mal-drenado. Em excesso, o fator umidade do solo faz com que as plantas aumentem o ciclo estimulando o crescimento vegetativo e prejudicando o desenvolvimento reprodutivo; assim, em regiões com alagamento do solo e em anos com elevadas precipitações as leituras de CEa possuem comportamento inverso ao da produtividade do algodoeiro. Anos com precipitação em excesso ou de estiagem tendem a intensificar a variabilidade da produtividade (Vieira et al., 2010). Segundo Amado et al. (2009) somente com a avaliação espacial e temporal dos dados de colheita é que se encontrar parâmetros para determinação de unidades de manejo diferenciado das culturas.

$\mathrm{O}$ pH $\mathrm{H}_{2} \mathrm{O}$ apresentou a maior correlação com a CEa na camada superficial do solo (0-0,3 $\mathrm{m})$, com $\mathrm{r}=0,60$. Também possível verificar correlação positiva entre CEa medida de $0-0,3 \mathrm{~m}$ com a saturação por bases, $\mathrm{K}$ trocável, soma de bases trocáveis e Ca trocável, de (valor de r) 0,56, 0,48, 0,48 e 0,46, respectivamente. Pelas relações estabelecidas a condutividade elétrica pode ser uma boa ferramenta para identificar a variabilidade de acidez e fertilidade no solo (Molin \& Rabello, 2011; Zonta et al.,2014).

O estudo de Mooney et al. (2010) em lavouras de algodão em 16 estados, nos EUA, indica que a adoção de ferramentas de AP ocorre em $63 \%$ dos produtores porém apenas $2 \%$ utilizam o monitoramento da produtividade. No entanto, entre todas as tecnologias de AP utilizadas os mapas de colheita foram os que mais auxiliaram na tomada de decisão atingindo $28 \%$ dos casos (Torbett et al., 2007). Entre os principais fatores que definem a lucratividade de uma cultura estão a produtividade e os custos de produção sendo que a maximização da eficiência de uso dos recursos disponíveis permite a sustentabilidade econômica do sistema produtivo. Neste estudo a utilização de mapas de produtividade no algodoeiro, espacial e temporalmente, possibilitou identificar e delimitar zonas homogêneas com problemas de excesso de água no solo possibilitando planejar variedades ou culturas tolerantes ao excesso de água no solo. As áreas com baixos níveis de nutrientes no solo poderão ser corrigidas (Zonta et al., 2014) e a adubação ajustada conforme o potencial produtivo das diferentes zonas, principalmente se os padrões de produtividade forem confirmados pela produtividade de outras culturas

\section{Conclusões}

1. A utilização de mapas de produtividade no algodoeiro permitirá investigar a variabilidade existente nas lavouras devido à quantidade e à qualidade dos dados obtidos pelos sensores das colhedoras.

2. Pela condutividade elétrica aparente é possível identificar variabilidade da fertilidade do solo pelas relações obtidas com $\mathrm{pH} \mathrm{H}_{2} \mathrm{O}, \mathrm{pH} \mathrm{CaCl}$, soma de bases, saturação por bases, $\mathrm{Ca}, \mathrm{Mg}$ e $\mathrm{K}$ trocáveis e correlação negativa com $\mathrm{H}+\mathrm{Al}$.

3. A combinação de atributos de solo associados à produtividade do algodoeiro possibilita estabelecer zonas homogêneas que podem ser tratadas com as ferramentas de AP. 
4. A adição de doses crescentes de P possibilitou o incremento da produtividade do algodoeiro, o que pôde ser validado espacialmente pelo mapa de produtividade.

\section{Agradecimentos}

À Fazenda Pamplona e à SLC Agrícola, que possibilitaram a realização deste trabalho; à Universidade Federal do Rio Grande do Sul e ao Programa de Pós- Graduação em Ciência do Solo, pela oportunidade de frequentar o curso de Mestrado e à Embrapa Algodão, pela utilização do protocolo experimental, base deste trabalho, e em especial à pesquisadora Ziany Neiva Brandão, pela constante colaboração.

\section{Literatura Citada}

Amado, T. J. C.; Pes, L. Z.; Lemainski, C. L.; Schenato, R. B. Atributos químicos e físicos de latossolos e sua relação com os rendimentos de milho e feijão irrigados. Revista Brasileira de Ciência do Solo, v.33, p.831-843. 2009.

Amado, T. J. C.; Pontelli, C. B.; Santi, A. L.; Viana, J. H. M.; Sulzbach, L. A. de S. Variabilidade espacial e temporal da produtividade de culturas sob sistema plantio direto. Pesquisa Agropecuária Brasileira, v.42, p.1101-1110, 2007.

Blackmore, B. S.; Godwin, R. J.; Fountas, S. The analysis of spatial and temporal trends in yield map data over six years. Biosystems Engineering, v.84, p.455-466, 2003.

Cambardella, C. A.; Moorman, T. B.; novak, J. M.; Parkin, T. B.; Karlen, D. L.; Novak, J. M. T.; Konolka, A. E. Field-scale variability of soil properties in Central Iowa Soils. Soil Science Society of America Journal, v.58, p.1501-1511, 1994.

Cerri, D. G. P.; Magalhães, P. S. G. Correlation of physical and chemical attributes of soil with sugarcane yield. Pesquisa Agropecuária Brasileira, v.47, p.613-620. 2012.

Deere, J. Colhedora de algodão John Deere. Base de dados. <http://www.deere.com.br/pt_BR/ag/products/ cottonpicker_model_1.html> 2 Set. 2012.

EMBRAPA - Embrapa Solos. Manual de análises químicas de solos e fertilizantes. Brasília: Embrapa Informação Tecnológica; Brasília: Embrapa Solos, 2009. 627p.

EMBRAPA - Empresa Brasileira de Pesquisa Agropecuária. Centro Nacional de Pesquisa de Solos. Sistema Brasileiro de Classificação de Solos. Brasília: EMBRAPA, 2013. 353p.

Guo, W.; Maas, S. J.; Bronson, K. F. Relationship between cotton yield and soil electrical conductivity, topography, and landsat imagery. Precision Agriculture, v.13, p.678-692. 2012.

Konopatzki, M. R. S.; Souza, E. G.; Nóbrega, L. H. P.; UribeOpazo, M. A.; Suszek, G. Spatial variability of yield and other parameters associated with pear trees. Engenharia Agrícola, v.32, p.381-392. 2012.

Lark, R. M.; Bolan, H. C.; Mayr, T.; Bradley, R. I.; Burton, R. G. O.; Dampney, P. M. R. Analysis of yield maps in support of field investigation of soil variation. In: European Conference on Precision Agriculture, 2, 1999. Odense. Proceedings... Odense: Denmark Sheffield Academic Press, 1999. p.151162.
Menegatti, L. A. A.; Molin, J. P. Remoção de erros em mapas de produtividade via filtragem de dados brutos. Revista Brasileira de Engenharia Agrícola e Ambiental, v.8, p.126134, 2004.

Michelan, R.; Souza, E. G. de.; Uribe-Opazo, M. A. Determinação e remoção do tempo de atraso em mapas de colheita de milho. Acta Scientiarum Agronomy, v.29, p.147-155, 2007.

Milani, L.; Souza, E. G. de; Uribe-Opazo, M. A.; Gabriel Filho, A.; Johann, J. A.; Pereira, J. O. Unidades de manejo a partir de dados de produtividade. Acta Scientiarum Agronomy, v.28, p.591-598, 2006.

Montezano, Z. F.; Corazza, E. J.; Muraoka, T. Variabilidade especial da fertilidade do solo em área cultivada e manejada homogeneamente. Revista Brasileira Ciência do Solo, v.30, p.839-847, 2006.

Molin, J. P.; Rabello, L. M. Estudos sobre a mensuração da condutividade elétrica do solo. Engenharia Agrícola, v.31, p.90-101, 2011.

Mooney, D. F.; Roberts, R. K.; English, B. C.; Lambert, D. M.; Larson, J. A.; Velandia, M.; Larkin, S. L.; Marra, M. C.; Martin, S. W.; Mishra, A.; Paxton, K. W. Rejesus, R.; Segarra, E.; Wang, C.; Reeves, J. M. Precision farming by cotton producers in twelve southern states: Results from the 2009 southern cotton precision farming survey. Knoxville: University of Tennessee, Department of Agricultural and Resource Economics. 2010. 70p. Research Reports 91333

Rosolem, C. A.; Echer, F. R.; Lisboa, I. P.; Barbosa, T. S. Acúmulo de nitrogênio, fósforo e potássio pelo algodoeiro sob irrigação cultivado em sistemas convencional e adensado. Revista Brasileira de Ciência do Solo, v.36, p.457-466, 2012. Sousa, D. M. G.; Lobato, E.; Rein, T. A. Adubação com fósforo. In: Sousa, D. M. G.; Lobato, E. (ed.). Cerrado: Correção do solo e adubação. Planaltina: Embrapa Cerrados. 2004. p.147-168.

Souza, J. G.; Beltrão, N. E. de M.; Santos, J. W. dos. Fisiologia e produtividade do algodoeiro em solo encharcado na fase de plántula. Pesquisa Agropecuária Brasileira, v.36, p.425430, 2001.

Torbett, C. J.; Roberts, R. K.; Larson, J. A.; English, B. C. Perceived importance of precision farming technologies in improving phosphorous and potassium efficiency in cotton production. Precision Agriculture, v.8, p.127-137. 2007.

Vanni, S. M. Modelos de regressão: estatística aplicada. São Paulo: Legmar Informática, 1998. 177p.

VERIS Technologies. 2011.<http://www.veristech.com>. 2 Set. 2012.

Vieira, S. R.; Guedes Filho, O.; Chiba M. K.; Mellis, E. V.; Dechen, S. C. F.; Maria, I. C. de. Variabilidade espacial dos teores foliares de nutrientes e da produtividade da soja em dois anos de cultivo em um Latossolo Vermelho. Revista Brasileira de Ciência do Solo, v.34. p.1503-1514, 2010.

Vieira, S. R. Geoestatística em estudos de variabilidade espacial do solo. In: Novais, R. F.; Alvarez V., V. H.; Schaefer, G. R. (ed). Tópicos em ciência do solo. Viçosa: Sociedade Brasileira de Ciência do Solo, v.1, p.1-54, 2000. 
Walton, J. C.; Lambert, D.M.; Roberts, R. K., Larson, J. A., English, B. C.; Larkin, S. L.; Martin, S. W.; Marra, M. C.; Paxton, K. W.; Reeves, J. M. Adoption and abandonment of precision soil sampling in cotton production. Journal of Agricultural and Resource Economics, v.33, p.428-448, 2008.
Zonta, J. H.; Brandão, Z. N.; Medeiros, J. da C.; Sana, R. S.; Sofiatti, V. Variabilidade espacial do solo em área cultivada com algodoeiro no Cerrado do Brasil. Revista Brasileira de Engenharia Agrícola e Ambiental, v.18, p.595-602, 2014. 